\title{
Análisis del mercado de valores como alternativa de financiamiento para las PYMES en Latinoamérica'
}

\author{
Stock market analysis as a financing alternative for SMEs in Latin \\ America
}

\author{
Jairo Beymar Céspedes López ${ }^{2}$ \\ Remy Silvia Martinez Pairo ${ }^{3}$
}

\section{RESUMEN}

En Latinoamérica las pequeñas y medianas empresas (pymes) se encuentran en constante crecimiento, y para financiarlo requieren de recursos de terceros, la forma tradicional de hacerlo es a través de la banca, sin embargo, existe otra alternativa atractiva en el sistema, la cual es el mercado de valores, en su sector tanto de renta variable como de renta fija, y los mercados alternativos bursátiles existentes en la región. El presente artículo analiza la situación latinoamericana de las pymes y sus mercados de valores, su percepción del financiamiento, sus beneficios y razones de hacer, o no uso de esta alternativa estratégica. Este análisis se realiza en función de los datos reales de mercado y asumiendo una posición crítica y propositiva de la alternativa al financiamiento empresarial en la región.

\footnotetext{
1 El presente artículo nace como resultado de observar el crecimiento continuo de los mercados de capitales en el mundo, además del crecimiento empresarial en Latinoamérica. Representa un artículo de reflexión.

2 Ingeniero Financiero, Universidad Católica Boliviana "San Pablo", Cochabamba, Bolivia. Maestrando en Instituciones y Mercados Financieros de la Universidad Complutense de Madrid y su centro adscrito CUNEF, Madrid, España. Ex Consultor en Rediseño Curricular y Docente Pregrado, Universidad Católica Boliviana “San Pablo”, Cochabamba, Bolivia. Miembro del Comité Científico de la Revista "Ingeniería Industrial: Actualidad y Nuevas Tendencias" de la Universidad de Carabobo, Venezuela. Dirección postal: Calle Manuel Arauco, Cochabamba, Bolivia. Correo Electrónico: jairocespedeslopez@gmail.com

3 Magíster en Dirección y Gestión Financiera, Universidad Rey Juan Carlos y su centro adscrito EAE Business School, Madrid, España. Magister en Gestión Financiera, Universidad Mayor de San Andrés, La Paz, Bolivia. Economista, Universidad Mayor de San Andrés, La Paz, Bolivia. Analista, Ministerio de Economía y Finanzas Públicas, La Paz, Bolivia. Dirección postal: Calle Rene Dorado, La Paz, Bolivia. Correo electrónico: remy.martinez777@gmail.com

(c) Los autores. Este artículo es publicado por Pensamiento Crítico de la Facultad de Ciencias Económicas, Universidad Nacional Mayor de San Marcos. Este es un artículo de acceso abierto, distribuido bajo los términos de la licencia Creative Commons Atribucion - No Comercia_Compartir Igual 4.0 Internacional. (http://creativecommons.org/licenses/by-nc-sa/4.0/) que permite el uso no comercial, distribución y reproducción en cualquier medio, siempre que la obra original sea debidamente citada.
} 
Palabras clave: Financiamiento; mercado de valores; finanzas; empresas. Clasificación JEL:G15, G30, M10, M13

\section{ABSTRACT}

In Latin America, Small and Medium Enterprises (SMEs) are in constant growth, and to finance it they require resources from third parties, the traditional way of doing it is through banking, however, there is another attractive alternative in the system, which it is the stock market, in its sector of both variable income and fixed income, and the alternative stock markets existing in the region. This article analyzes the Latin American situation of SMEs and their stock markets, their perception of financing, their benefits and reasons for doing, or not using this strategic alternative. This analysis is made based on real market data and assuming a critical and proactive position of the alternative to business financing in the region.

Keywords: Financing; Stock market; finance companies.

\section{Introducción}

Las empresas son el motor de la economía de un país, en el caso de Latinoamérica, según la Agencia EFE, las pequeñas y medianas empresas (pymes) aportan al Producto Interno Bruto (PIB) regional en aproximadamente un $30 \%$, contribuyendo de esta manera con la generación de empleos para la población, fomentando el crecimiento y por ende el desarrollo de los países, mejorando así la calidad de vida de la sociedad.

De esta manera, es relevante que una empresa obtenga liquidez para realizar sus actividades operativas, financieras, administrativas y comerciales, para así continuar operando y ampliar su negocio constantemente. Una empresa puede financiarse con recursos internos o externos, los primeros tienen limitaciones, puesto que los dueños pueden disponer solamente de sus recursos propios como capital social o depender de los recursos que se generen en el negocio tales como reservas.

Por consiguiente, a través de la financiación externa se puede obtener recursos de terceros que estén dispuestos a invertir en la empresa a cambio de un crédito financiero, ya sea a través de un mercado bancario, mercado de valores, crowdlending ${ }^{4}$ o el sector público; al respecto, usual-

4 El crowdlending, es una forma de financiamiento que es pactada de manera directa entre las empresas nuevas y los inversores, usualmente se realiza a través de una plataforma on line y la utilizan las empresas start up. 
mente se utiliza la primera opción, dejando de lado el resto de las fuentes que no son tradicionales, y aún en ésta, las pymes tienen problemas para acceder al capital.

En el caso de Latinoamérica, las empresas son heterogéneas en capacidad productiva, innovación, tecnología y en recursos humanos, razón por la cual es más difícil que logren alcanzar un nivel de productividad que sea competitivo internacionalmente, por esta razón no logran exportar y así ampliar su mercado.

Los recursos obtenidos por una empresa pueden ser utilizados: ya sea para iniciar sus actividades por primera vez, iniciar un nuevo proyecto; o para ampliar el negocio, con una mayor capacidad productiva consecuencia de implementar nueva tecnología, innovación en los procesos, especialización de los recursos humanos, etc.

En ese contexto, en Latinoamérica se presenta una gran cantidad de emprendimientos, los cuales no logran desarrollarse debido a la falta de financiamiento, muchos de ellos inician operaciones, pero al transcurrir un corto lapso de tiempo son incapaces de conseguir los recursos necesarios para mantenerse en el mercado, lo cual conlleva el cese de operaciones y posteriormente el abandono del proyecto.

Asimismo, las empresas ya establecidas tienen como siguiente objetivo el de expandirse, naturalmente cuando una empresa comienza a progresar deja de ser Pyme y se convierte en una grande empresa, por lo que la necesidad de encontrar liquidez se hace más latente.

Por las razones expuestas, es imprescindible que una empresa consiga financiamiento para llevar a cabo sus actividades y en efecto exponen; y una de las alternativas para acceder al capital es el mercado de valores.

\section{Metodología}

En los siguientes acápites, se presenta una investigación de reflexión, realizada con el objeto de mostrar el mercado de valores como alternativa de financiamiento para las pymes, se consideró a los países de Latinoamérica. 
Se inicia con un análisis, sobre la importancia de las empresas en la región, la forma en la que formularían su estructura de capital considerando principalmente el costo. Asimismo, se analiza el comportamiento de la región en cuanto al mercado de valores, además se observan las razones por las cuales dichas empresas tienen dificultades para lograr el financiamiento para sus operaciones, sobre todo en la banca tradicional.

Una vez determinada la importancia de las pymes en la economía de la región, se exponen las ventajas sobre el acceso al financiamiento a través del mercado de valores, como una alternativa a la banca tradicional, con el objeto de fomentar el crecimiento de las empresas.

Para lo cual, se utilizan técnicas de descripción y análisis de datos como base, aplicándose una investigación documental, empleando la revisión de bibliografía académica y de entidades locales y regionales que han estudiado el sector de las pymes con anterioridad, por último, se expone los resultados de un análisis con criterio personal sobre la situación de este tópico en Latinoamérica.

\section{Marco teórico}

El presente acápite, plasma de manera teórica la importancia de las pymes en la región y su relación con el mercado de valores, con el objeto de ilustrar los beneficios que se logran a través de la diversificación de mercados. Por lo cual, en primera instancia se especifica teóricamente la parte financiera empresarial, para posteriormente entrar en tema de mercados financieros como alternativa dentro de la estructura de capital de estas empresas.

\subsection{Pymes}

Para entrar en tema, es importante resaltar la importancia del crecimiento de las pymes en la región, siendo uno de los factores más relevantes la participación en la generación de empleo por parte de las mismas, la cual se muestra a continuación en el gráfico 1.

Del gráfico se deduce que, las micro, pequeñas y medianas empresas (mi pymes) en su conjunto generan un 61\% del empleo formal en Latinoamérica, este es un aporte para las economías, puesto que, al generarse 
empleo se garantiza la dotación de ingresos a las familias, lo cual fomenta el consumo y por ende la producción de bienes y servicios.

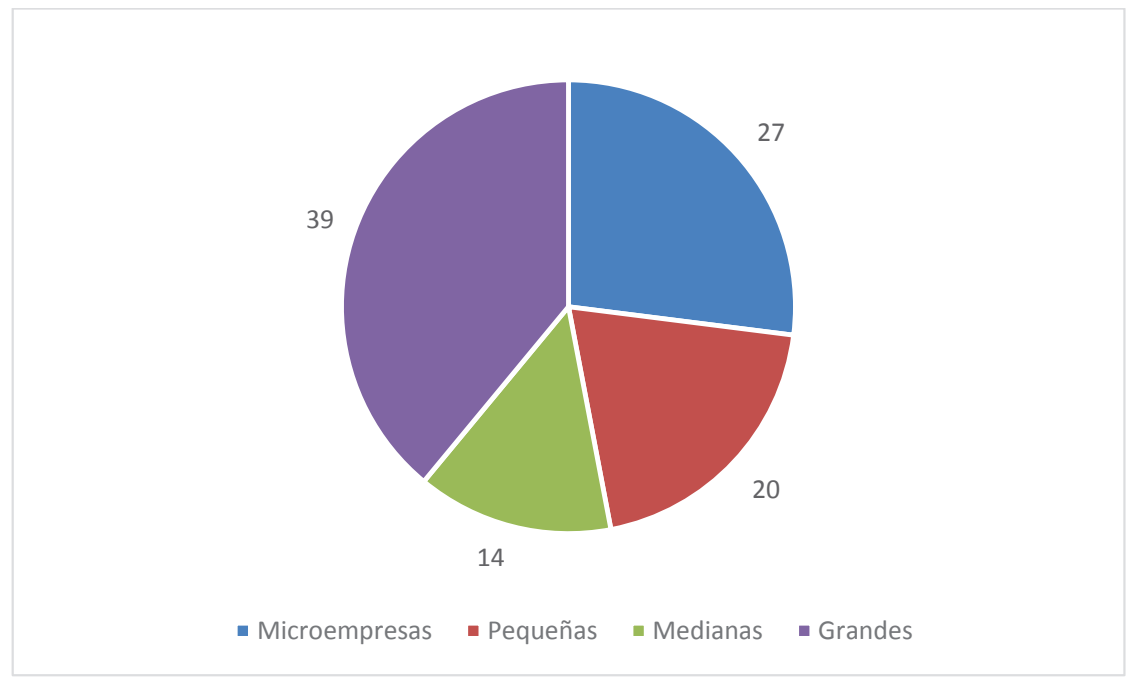

Gráfico 1. Participación de las empresas en el empleo en Latinoamérica Fuente: MIPYMES en América Latina

De la misma manera, la participación de las mipymes en la producción y las ventas representan el 15\% en la región, tal como se muestra en el gráfico 2 .

Por otro lado, CEPAL(2018) indican que en América Latina las microempresas representan el $88 \%$ de las empresas formales, aportan el $27 \%$ del empleo y apenas el 3,2\% de la producción, mientras que, en la Unión Europea las microempresas constituyen el 93\% de las empresas, el $30 \%$ del empleo y el 20\% de la producción. Esta situación refleja las características de las microempresas (y de alguna forma de las pymes también) de la región, es decir empresas que operan en mercados locales acotados, que dependen de la evolución de la demanda interna, en sectores con bajas barreras a la entrada y salida, con tasas elevadas de natalidad y mortalidad y que, muchas veces, responden más a estrategias de autoempleo y sobrevivencia que a una dinámica de desarrollo empresarial.

Entonces según CEPAL (2018), por un lado, las mipymes representan la enorme mayoría de las empresas y el empresariado de la región, apor- 
tando significativamente a la generación de empleo, pero por el otro, la productividad de las pymes es baja, y extremadamente baja en el caso de las microempresas, esto pone en evidencia la importancia del sector y las carencias que requieren ser subsanadas.

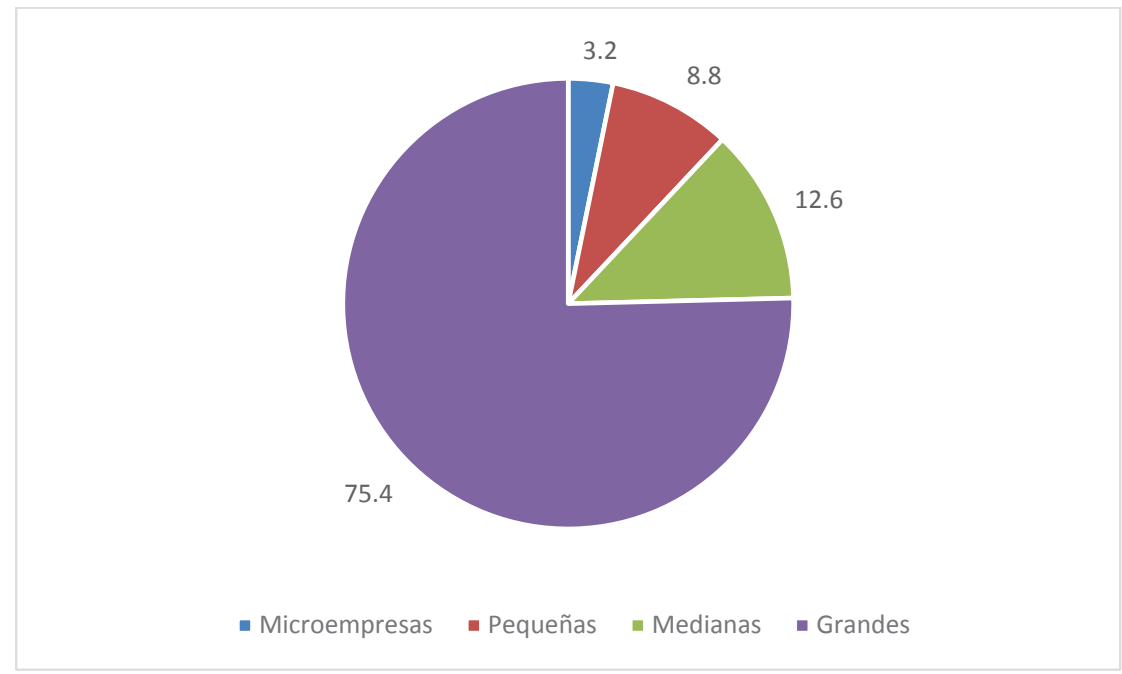

Gráfico 2. Participación de las empresas en la producción y las ventas Fuente: MIPYMES en América Latina

Igualmente, en todos los casos la inserción de las mipymes (en particular las microempresas y las pequeñas empresas) en la economía se realiza en las ramas de menor productividad, en las cuales hay bajas barreras a la entrada y donde la necesidad y los incentivos para operar en conexión con otras empresas (generando redes o clúster) son muy bajos, y por lo tanto también son reducidas las posibilidades de generar economías externas que incrementen la especialización (y de la fuerza laboral), la propensión a innovar y la productividad, esto también dificulta la sobrevivencia y el crecimiento de las empresas.

De igual manera, CEPAL (2018) argumentan que la estructura productiva de los países de la región, en la cual predominan las grandes empresas especializadas en sectores intensivos en recursos naturales y algunos servicios de alta intensidad de capital, hace que no haya incentivos para que las mipymes puedan insertarse en actividades de mayor valor agregado en la región. 
Es así, que las pymes son igual de importantes que las grandes empresas en una economía regional, principalmente por la generación de empleo formal que aportan éstas a los países y por el potencial de crecimiento que tienen a pesar de las limitaciones que puedan existir.

\subsection{Finanzas en las pymes}

Todas las empresas necesitan de capital para introducirse dentro de un mercado, sea cual sea este, y para ello deben acudir a financiar sus operaciones, para lo cual, existen distintas maneras de hacerlo. Según Van denVerghe (2015, p.225), se presentan dos fuentes de financiamiento, una de ellas es la financiación interna o autofinanciación que es "generada por las operaciones propias de la empresa, mediante el no reparto de utilidades, la creación de reservas para un fin específico o por el descuento de documentos generados por las operaciones normales de la compañía."

Por otro lado, Van den Verghe (2015, p. 225) indica que la financiación externa "comprende los fondos captados por fuera de la empresa, aportes de los socios antiguos, la inclusión de nuevos propietarios, la emisión de acciones o bonos, cartas de crédito, leasing, factoring, líneas de crédito bancario, fondos de garantía, tarjetas de crédito y financiación en divisas (préstamos del exterior)."

Es así, que las empresas se van consolidando financieramente, ya sea con una fuente de recursos interna o externa, y a efecto de definir el tipo de financiación que utilizará se analiza la estructura de capital de la empresa, la cual responde a las características del sector de dicha empresa, y a los objetivos de largo plazo plasmados por la misma.

De esta manera, según Álvarez (2016, p.149) la estructura de capital es "la combinación del pasivo a largo plazo y el capital común o patrimonio de los accionistas, que permite a las empresas ejecutar sus inversiones, crecer, ser rentables en sus operaciones y crear valor.", es decir, cuánto se ha de financiar con recursos de una fuente interna, que puede ser capital introducido por los accionistas o duelos, y cuánto de recursos se conseguirán a través de financiación externa.

Sin embargo, el uso de capital supone al mismo tiempo la elaboración de un presupuesto de capital, mismo que Van den Verghe (2015, p. 225) 
indica que "es el proceso de planear y administrar las inversiones a largo plazo por parte de la empresa. Mediante la elaboración del presupuesto de capital, debe identificar las oportunidades de inversión, evaluando la magnitud, el tiempo y el riesgo de los futuros flujos de efectivo, con el fin de evitar la iliquidez de la empresa.", esto pone en evidencia los lineamientos y políticas que la empresa aplicará a largo plazo.

Este aspecto es fundamental para los inversionistas, ya que, para financiar las actividades de la empresa, requieren conocer la composición del Balance General en cuanto a sus activos, pasivos y patrimonio, es decir, la estructura de capital, con el objeto de considerar la estabilidad de la empresa, los rendimientos que genera, para así tener la confianza necesaria para inyectar su capital a cambio de una recompensa.

Una vez expuesto esto, Álvarez (2016, p. 149) menciona que es de relevancia tener en cuenta la estructura financiera, que "está compuesta por la totalidad del lado derecho del balance, pasivos a corto y largo plazo y patrimonio, y refleja cómo financia la empresa todos sus activos y operaciones." dicha composición de la estructura de capital de una empresa se muestra a continuación en la tabla 1:

Tabla 1

Estructura de capital

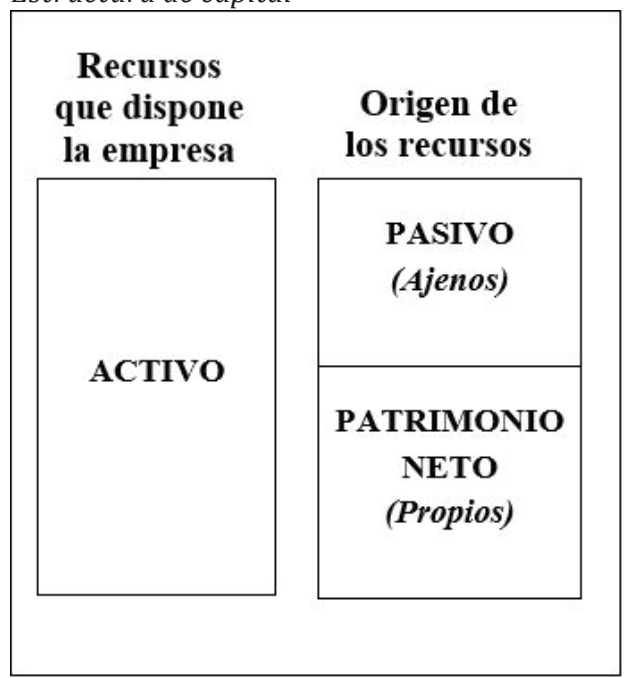

Fuente: Enciclopedia Financiera 
En base a dichos conceptos, y una vez realizado el análisis de la estructura de capital se determina el tipo de financiamiento pertinente, en ese sentido Álvarez(2016, p. 149) indica que una buena estrategia "es diferenciar el costo y la rentabilidad del dinero invertido -propio y ajeno- en las empresas y establecer los parámetros de rentabilidad de sus actividades económicas y de inversión. Esta diferencia permite a los ejecutivos desarrollar estrategias que optimicen las inversiones, maximicen la riqueza y creen valor en las empresas.", por lo cual es importante tener bien definida la estructura de capital para evaluar cuál es el financiamiento adecuado para las empresas, según sus características y necesidades.

\subsection{Sistema financiero y mercado}

Con la finalidad de establecer la estructura de capital y determinar las fuentes de financiamiento de una empresa, se evalúa los costos de cada una de ellas, procurando lograr una diversificación apropiada para la empresa, para lo cual se acude al sistema financiero.

De esta manera, la Guía de Mercado de Valores de Colombia (2014, p.21), indica que "El sistema financiero es el conjunto de instituciones y operaciones a través de las cuales se transfieren recursos disponibles para depositar o invertir (sector superavitario), a las empresas que los requieren para sus proyectos (sector deficitario). El sistema financiero comprende el mercado bancario, también llamado intermediado, y el mercado de valores, también llamado desintermediado."

Entonces un sistema financiero, es el conjunto de instituciones que realizan operaciones financieras de manera directa o indirecta, facilitando el intercambio de aquellos entes que tienen un excedente monetario con los entes que lo necesitan, a través de los intermediarios financieros, rigiéndose bajo las normas vigentes establecidas.

El sistema financiero se compone principalmente por el mercado monetario y el mercado de capitales. Éste último denominado también mercado de valores, según Caixabank, (2012, p.15):

En torno al mercado de valores se mueven los inversores u oferentes de capital (personas físicas o jurídicas) que acuden a colocar su dinero en valores emitidos por los demandantes de capital (empresas, instituciones públicas, etc.). Junto a estos elementos personales están quienes actúan como intermediarios financieros en el 
mercado y cuya función profesional es facilitar los recursos necesarios para poner en relación a oferentes y demandantes.

Es decir que, el mercado de valores tiene como principal función facilitar el flujo de ahorro e inversión de manera directa, y fundamentalmente se utiliza para el financiamiento de mediano y largo plazo de las empresas.

En dicho mercado, los principales actores son: los emisores que pueden ser instituciones privadas o públicas que buscan financiarse a través de títulos valores; los inversores personas o instituciones nacionales o extranjeras que tienen un excedente de recursos, las agencias de bolsa, encargadas de estructurar las emisiones de los títulos valores; las calificadoras de riesgo, quienes evalúan el nivel de riesgo que tiene el emisor considerando toda la información disponible sobre la misma; la bolsa de valores como ente que facilita la negociación de los valores y un ente supervisor que supervise el cumplimiento de la norma vigente establecida para operar en este tipo de mercado.

A su vez, Caixabank (2012, p.15) indica entre otras cosas, el mercado primario y el mercado secundario, argumentando sobre el primero que "es aquel en el que se desarrolla la oferta y demanda de valores en el momento de la entrada de una sociedad en bolsa, una privatización de empresa pública o de la ampliación de su capital con la aportación de nuevos títulos, y que requiere una relación directa entre el emisor y el suscriptor de los valores."

Por otro lado, el mercado secundario, de igual manera Caixabank (2012, p. 15) indica que este "es aquel en el que se produce la confrontación de ofertas y demandas de valores ya emitidos con anterioridad y admitidos a negociación en bolsa."

De esta forma, en el mercado primario, la empresa la emisora, ofrece una tasa de interés a cambio de los recursos erogados; y el mercado secundario hace referencia al espacio donde los valores son negociados por segunda o más veces por el poseedor del título bajo diferentes condiciones que en el mercado primario.

Con relación a los activos financieros, Heras (2001, s/p) indica lo siguiente: "Es un contrato que concede al inversor, ciertos derechos que en el futuro deberían ser satisfechos con cargo al emisor del mismo; por tan- 
to, es un medio de mantener riqueza para quien lo posee y un pasivo para quien lo genera."

De este modo, los títulos valores emitidos en la bolsa de valores, pueden ser de renta fija o renta variable, el primero se refiere a aquellos títulos que ofrecen una renta fija, es decir que independientemente del comportamiento que tenga el título, el rédito a ser percibido por el inversionista es fijo, el título generalmente emitido, es el bono; en el segundo caso la ganancia obtenida por el inversionista dependerá del comportamiento del título valor en el mercado, principalmente está referida a la emisión de acciones.

Por último, todo esto se realiza con el fin de que exista una adecuada gestión estratégica financiera, al respecto Álvarez (2016, p. 160) argumenta que "la administración del financiamiento consiste en seleccionar óptimamente recursos financieros que necesita la empresa a fin de aprovechar las oportunidades de permanecer en el mercado y contribuir a su crecimiento.", por lo tanto, con la gestión financiera se busca la administración óptima de los recursos aprovechando el mercado.

\subsection{Financiación en la actualidad}

Opertti (2017) indica que las pymes son fundamentales para el ecosistema productivo, ya que representan el 99\% de las empresas de América Latina y el Caribe y generan el 67\% del empleo de la región. Sin embargo, todas las empresas de la región enfrentan problemas en común, como ser la desconexión con los mercados, la falta de oportunidades de financiamiento debido a distintos factores, esto entre otras cosas impide el crecimiento y desarrollo de las empresas.

Por otro lado, de acuerdo a Rojas (2017) en 2010 América Latina y el Caribe tenía el porcentaje más bajo de empresas con una restricción financiera total del 9\% entre los mercados emergentes; sin embargo, tenía el segundo porcentaje más alto de empresas con una restricción parcial, esto quiere decir que las empresas acudían a fuentes distintas a los bancos y otros intermediarios financieros para el capital de trabajo, como el crédito de proveedores. 
Según OCDE (2015), algunos países en el mundo utilizan estrategias de mecanismo anti cíclico, por ejemplo, el gobierno de Gran Bretaña en 2014 se encargó de la creación de una nueva institución, el British Business Bank, con la finalidad de crear mayor opción de financiamiento a las pymes. Por otro lado, existen instituciones similares, existentes en países como Francia, Portugal, Tailandia y la República Checa, entre otros. Además de ofrecer créditos y garantías, las agencias especializadas disponen de una variedad de productos para las pymes, las cuales incluyen cuentas corrientes, tarjetas de crédito, sistemas de pago electrónico (para clientes, proveedores y de servicios), leasing, factoring, etc., todo ello con el objeto de establecer mecanismos que permitan el desarrollo de las empresas.

En el otro lado de la moneda, existen problemáticas o barreras para el desarrollo de las empresas en la región, los cuales se muestra gráficamente a continuación en el gráfico 3:

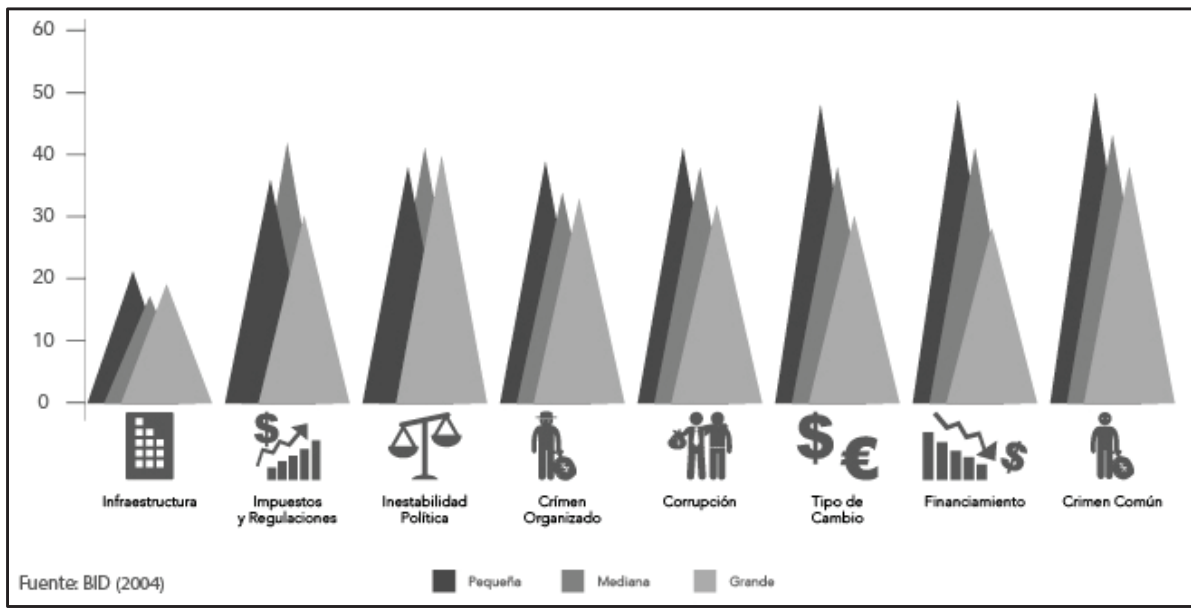

Gráfico 3. Obstáculos para el desarrollo de pymes en América Latina

Entre los diversos problemas que aquejan a las empresas, Rojas (2017) resalta que uno de los problemas más latentes es la dificultad de acceder al financiamiento, el cual se refleja en la tendencia a la baja de la proporción del crédito total que ha sido otorgado a las pymes a lo largo de los años, pues 
este porcentaje de financiamiento representa menos del $50 \%$ en todos los países, así pues, el promedio para Latinoamérica alcanza solo el 12\%.

Del mismo modo, un estudio realizado por la CEPAL (2010) indica que, en países como Brasil, Bolivia, Costa Rica, Panamá, El Salvador y México, las altas tasas de interés y la insuficiencia de garantías de las pymes son los factores más relevantes y los que más influyen en el uso de fuentes alternativas al crédito bancario para el financiamiento de estas empresas en la región.

Por consiguiente, Galán (2007) argumenta que es importante mencionar que, la problemática en Latinoamérica tiene entre otros, tres factores limitantes, como ser, las elevadas tasas de interés, la falta de acceso a créditos de largo plazo, y las dificultades asociadas a la constitución de garantías.

Cabe señalar que, la falta de garantías también es un factor relevante como barreras para el acceso a financiamiento y que con el propósito de superarlo se ha implementado un sistema de garantías; sin embargo, a pesar de todos los esfuerzos realizados, el alcance ha sido mínimo y el problema persiste, convirtiéndose en un gran reto a ser superado por los empresarios y los gobiernos de estos países, los cuales deberían tener como premisa impulsar a las pymes, debido a su importancia económica y social.

\section{Resultados y discusión}

En el presente acápite se muestra el desarrollo, resultado y discusión del tema principal, a partir de las bases teóricas y la realidad actual en Latinoamérica.

\subsection{Situación de las bolsas de valores en Latinoamérica}

Si bien las pymes han iniciado su inserción en el mercado de valores en los distintos países no solamente latinoamericanos, ya que cada vez tiende a ser más atractivo, es importante analizar el mismo considerando los actores de esta alternativa, siendo los fundamentales: los emisores, los inversionistas, los entes reguladores de cada mercado, y el mercado en sí mismo. 
Respecto de este último, cabe señalar que el objeto del presente análisis es el mercado de valores y su impacto en las empresas en Latinoamérica como fuente de recursos, es así que, tomando en cuenta que los países tienen una diversidad en cultura, políticas y economía, existen aquellos que llevan la delantera en la forma de obtener financiamiento mediante la bolsa de valores, sobre todo por los diversos instrumentos financieros que utilizan y la multiplicidad de operaciones que realizan. Para entrar en contexto, es imprescindible mencionar la situación de los mercados en la región.

En Latinoamérica, solamente se han registrado salidas a bolsa en Argentina, México, Chile y Perú durante 2017 y parte de 2018, probablemente esto depende de cuan atractivo es este mercado, y si es más conveniente salir a bolsa, o solamente emitir deuda, y para ello se procede a realizar el análisis de los datos de negociación tanto en mercado de renta variable como renta fija, datos que se muestran en la Tabla 2.

Tabla2

Negociación en renta variable en bolsas de Latinoamérica

\begin{tabular}{|c|c|c|c|c|c|c|c|c|}
\hline \multirow{2}{*}{ Bolsa } & \multicolumn{6}{|c|}{2018} & \multirow{2}{*}{$\begin{array}{c}\text { Acumulado } \\
2018\end{array}$} & \multirow{2}{*}{$\begin{array}{c}\text { Variación \% } \\
2017\end{array}$} \\
\hline & Julio & Agosto & Septiembre & Octubre & Noviembre & Diciembre & & \\
\hline Buenos Aires & 490 & 535 & 980 & 489 & 302 & 266 & 7,610 & 2.84 \\
\hline Boliviana & 0 & 0 & 1 & 1 & 1 & 7 & 282 & 200.00 \\
\hline Santiago & 3,485 & 2,791 & 2,945 & 3,631 & 2,682 & 6,821 & 55,119 & 50.42 \\
\hline Colombia & 1,099 & 1,111 & 1,231 & 1,514 & 995 & 716 & 15,060 & 8.00 \\
\hline Costa Rica & 3 & 8 & 3 & 7 & 2 & 8 & 52 & -10.63 \\
\hline Guayaquil & 3 & 2 & 2 & 2 & 2 & 1 & 35 & 64.23 \\
\hline Quito & 1 & 2 & 6 & 4 & 3 & 2 & 31 & 45.62 \\
\hline El Salvador & 2 & 0 & 2 & 1 & 0 & 0 & 11 & 200.00 \\
\hline Mexicana & 7,861 & 7,383 & 7,359 & 8,212 & 9,412 & 3,070 & 96,346 & -6.15 \\
\hline Panamá & 9 & 18 & 17 & 5 & 65 & 36 & 326 & -31.17 \\
\hline Lima & 196 & 198 & 406 & 271 & 429 & 680 & 4,744 & -32.10 \\
\hline Caracas & 56 & 2 & 2 & 11 & 5 & 2 & 204 & -99.69 \\
\hline
\end{tabular}

Fuente: Federación Iberoamericana de Bolsas (FIAB)

En el mercado de renta variable, se observa que países como Bolivia, Costa Rica, Ecuador (con las bolsas de Guayaquil y Quito) y El Salvador, son los que menor número de negociación muestran, si bien Bolivia pre- 
senta un monto más elevado que los otros países mencionados, esto se debe a una operación con un monto considerable de negociación en el mes de mayo de 2018, pero en realidad tiene muchos meses en los que no hay negociación, lo cual indica que el mercado de renta variable es realmente inactivo, y la salida a bolsa de una empresa con miras al financiamiento en este tipo de mercados es prácticamente nulo, debido a que no habría una negociación dinámica de sus acciones y su valor de mercado puede permanecer estático a pesar de tener un crecimiento de la empresa como tal.

A pesar de que países como: Costa Rica, Ecuador y El Salvador mantienen niveles bajos de negociación, al menos todos los meses del año presentan movimiento, lo cual es una buena señal de dinamismo del mercado, además como se mencionó anteriormente, el valor de mercado de las empresas que salen a bolsa es relevante en los mercados.

Por otro lado, Argentina, Brasil, Chile, Colombia, México y Perú son países que tienen altos montos de negociación en bolsa, esto implica que existen dinamismos en el mercado, sobre todo en los mercados de renta variable.

A continuación, en la Tabla 3 se muestra la negociación en renta fija:

Tabla 3

Negociación en renta fija en bolsas de Latinoamérica

\begin{tabular}{lccccccccc}
\multicolumn{1}{c}{ Bolsa } & & \multicolumn{1}{c}{$\mathbf{2 0 1 8}$} & \multicolumn{3}{c}{ Acumulado } & \multicolumn{2}{c}{ Variación \% } \\
& Julio & Agosto & Setiembre & Octubre & Noviembre & Diciembre & $\mathbf{2 0 1 8}$ & $\mathbf{2 0 1 7}$ \\
Buenos Aires & 8,994 & 9,573 & 12,590 & 8,197 & 7,448 & 8,527 & 118,271 & -7.25 \\
Boliviana & 1,739 & 1,531 & 1,501 & 1,527 & 1,165 & 1,153 & 16,370 & 34.78 \\
Santiago & 21,741 & 24,915 & 15,522 & 21,220 & 17,500 & 18,321 & 249,363 & 14.7 \\
Colombia & 29,216 & 33,552 & 26,869 & 22,418 & 20,421 & 16,578 & 333,329 & 7.16 \\
Costa Rica & 235 & 340 & 321 & 834 & 118 & 338 & 4,136 & -39.37 \\
Guayaquil & 15 & 23 & 78 & 65 & 112 & 44 & 547 & -26.35 \\
Quito & 34 & 47 & 44 & 68 & 110 & 31 & 536 & 26.03 \\
El Salvador & 82 & 214 & 96 & 89 & 30 & 21 & 998 & 61.35 \\
Mexicana & 1 & 1 & 2 & 3 & 6 & 1 & 39 & -66.77 \\
Panamá & 99 & 362 & 389 & 326 & 754 & 93 & 3,670 & 33.45 \\
Lima & 28 & 73 & 41 & 44 & 23 & 30 & 484 & 5.18 \\
Rep. Dominicana & 620 & 512 & 493 & 437 & 505 & 441 & 7,522 & -3.6 \\
\hline
\end{tabular}

Fuente: Federación Iberoamericana de Bolsas (FIAB) 
En la tabla precedente, aunque son mercados heterogéneos, se evidencia que todas las bolsas presentan una participación activa en la negociación a partir de activos de renta fija, sin embargo, algunas presentan mayor relevancia que otras. La Bolsa de Colombia presenta un mayor valor que el resto de la región y la Bolsa Mexicana de Valores refleja el valor más bajo de negociación en este tipo de activos, lo cual quiere decir que esta bolsa es más propensa a la renta variable que a la renta fija.

Por lo cual, lo que se puede evidenciar es que, al menos el mercado de renta fija funciona muy bien en toda Latinoamérica, lo cual es confortante, dado que lo que las empresas quieren en principio es conseguir financiación, y no una salida a bolsa como tal, hasta el momento de estar realmente preparadas para hacerlo.

En Latinoamérica, las bolsas funcionan de manera dinámica para el fin principal de las empresas, el financiamiento, en obviedad cada una con características diferentes, pero bajo un análisis general presentan un buen escenario para hacerlo.

\subsection{Iniciativa de integración financiera en Latinoamérica}

En Latinoamérica se está intentando lograr avances respecto a la forma de acceder al financiamiento bursátil, como muestra de ello se ha creado el denominado Mercado Integrado Latinoamericano (MILA), el cual está conformado por Chile, Colombia, México y Perú, quienes han marcado un hito en la historia de las operaciones financieras al conformar dicha alianza, la unión de los cuatro mercados de valores facilita la compra y venta de instrumentos de renta variable entre los brokers ${ }^{5}$ de dichos países, para así de esta manera generar un mayor acceso a los recursos disponibles en el mercado.

Según el MILA, los cuatro países representan el 38\% del PIB de América Latina y el Caribe, lo cual muestra la importancia de esta iniciativa para la región, esta integración ha negociado hasta junio de 2018 un monto de 590.721.419 \$US, de los cuales el país más representativo es México seguido de Chile.

5 Persona natural o jurídica que realiza compra y venta de instrumentos financieros según la solicitud de sus clientes, es un intermediario. 
Los más grandes emisores en 2018 fueron: Sociedad Química y Minera de Chile S.A., Ecopetrol de Colombia, NAFTRAC de México y Mi Banco de la Microempresa S.A. de Perú. Asimismo, las operaciones acumuladas del MILA a junio de 2018 ascienden a 27.998 y se puede invertir en más de 700 compañías de estos cuatro países.

Del mismo modo, los países integrantes crearon fondos para invertir en MILA, Chile tiene 5 fondos, Colombia 2, México 1 y Perú 2, los cuales totalizan un monto captado de 44.983.533 \$US.

Los principales índices que miden el desempeño del MILA son el S\&P

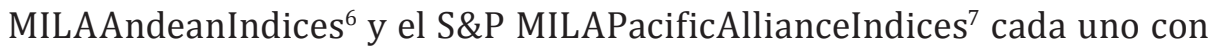
sus respectivos subíndices, los mismos fueron diseñados por Standard \& Poor's para medir el rendimiento de los mercados de acciones.

El primero está diseñado para medir el comportamiento de las 40 empresas más importantes y líquidas negociadas, además de evaluar el desempeño por sectores industriales; el segundo se enfoca en el universo de todas las acciones clasificadas según diversos aspectos.

Sin duda, esta iniciativa coadyuva con el impulso de la cultura financiera bursátil, convirtiéndose en un incentivo para que las empresas Latinoamericanas busquen en el mercado de valores el financiamiento para realizar sus operaciones con vistas a obtener recursos de cualquier fuente en el mundo.

\subsection{América Latina y su relación con el financiamiento a través de la bolsa como alternativa a la banca tradicional}

En los últimos años en el mundo entero, ha habido cambios en las finanzas, sobre todo como consecuencia de la crisis del 2008, la cual tuvo como

6 S\&P MILA AndeanIndices está compuesto por:

- S\&P MILA Andean 40 y

- S\&P MILA Andean Sector Indices (S\&P MILA Andean Financials y S\&P MILA Andean Resources)

7 S\&P MILA Pacific Alliance Indicesesta integrado por:

- S\&P MILA Pacific Alliance Composite

- S\&P MILA Pacific Alliance Select

- S\&P MILA Pacific Alliance Select ExREITs

- S\&P MILA Pacific Alliance Completion

- S\&P MILA Pacific Alliance Sector Indices

- S\&P MILA Pacific Alliance Size Indices 
impacto la salida de capitales de Latinoamérica; han pasado ya casi diez años, y se ha aprendido de los errores cometidos y planteado ajustes a la norma existente, cambios para mejorar el control del mercado y propuestas para hacerlo más eficiente, de esta manera el mercado de valores en las economías desarrolladas no solo es utilizado por los empresarios, si no también está empezando a ser conocido por el ciudadano de a pie, lo cual contribuye al desarrollo de su industria y economía.

En este contexto, es importante que las economías en desarrollo empiecen a utilizar los instrumentos financieros disponibles en el mercado de valores, sobre todo para que las empresas logren la liquidez necesaria para realizar sus operaciones y así ser competitivas a nivel mundial. El objetivo es lograr obtener recursos con los menores costos posibles, y sobre todo con costes menores a los de la fuente tradicional que es la banca, por lo cual, para observar el comportamiento de ambas fuentes de recursos se realiza una comparación de la profundización de cada una de ellas respecto del PIB.

A continuación, se presenta el Gráfico 4 que expone la comparación entre la proporción de los créditos y la capitalización bursátil respecto del PIB en Latinoamérica.

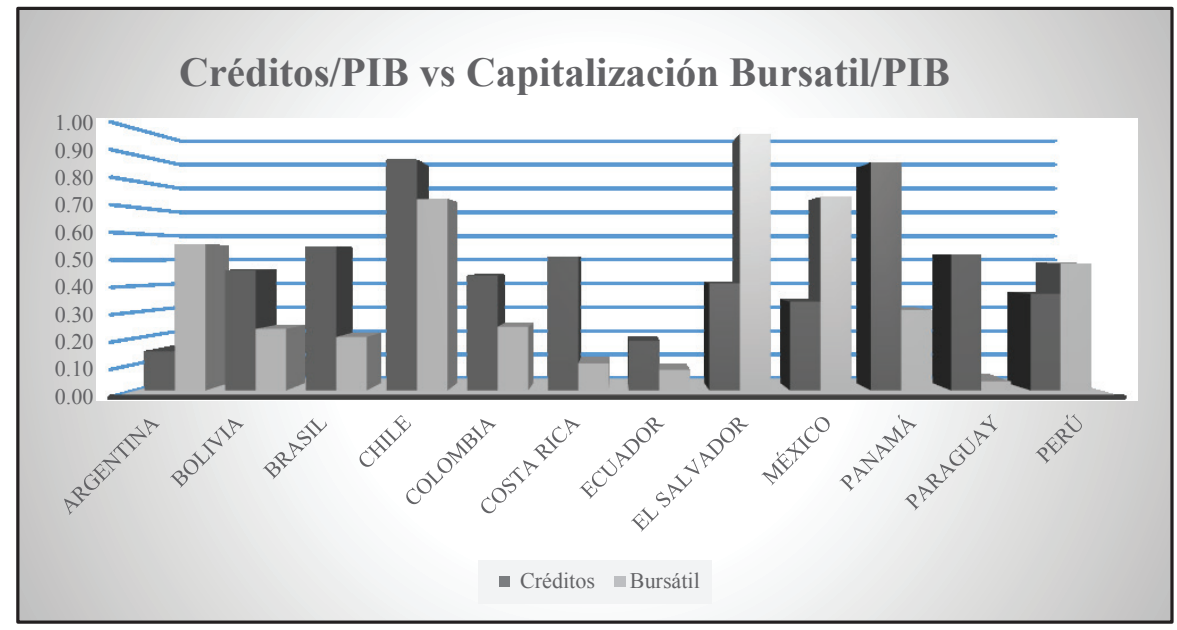

Gráfico 4. Créditos/PIB vs Capitalización Bursátil/PIB (2015)

Fuente: Elaborado en base a datos de la Federación Iberoamericana de Bolsas (FIAB), Federación Latinoamericana de Bancos (FELABAN) y Banco Mundial (BM) 
En base al gráfico 4 se observa que en cuanto a la profundización financiera, se observa que durante el periodo 2007-2015 en Latinoamérica, los países con más profundización, es decir los que más utilizaron el crédito bancario como fuente de financiación fueron: Chile, Panamá, Paragua y, Brasil, Costa Rica y Bolivia, siendo una limitación el hecho de que los bancos prestan montos bajos y cobran un alto interés por dicho préstamo, dejando de ser atractivo como fuente de financiamiento para los empresarios, pero además los productos y servicios ofrecidos no se ajustan a las necesidades y realidad de los clientes.

Por otro lado, respecto a la profundización bursátil se observa que los países que más participaron en el mercado fueron: Argentina, Chile, México, El Salvador y Perú, esto se debe principalmente al hecho de que las empresas que cotizan tienen un mercado internacional, lo cual les facilita el acceso a recursos.

En la gráfica, también se denota que la proporción de los créditos respecto del PIB es ascendente, esto implica que en los últimos años se ha estado utilizando el financiamiento ofertado por la banca en mayor proporción, esto ocurre principalmente debido a que los emprendedores no tienen conocimiento suficiente de la existencia de las alternativas a dicha financiación.

Asimismo, cuando se analiza la proporción de la capitalización bursátil respecto del PIB, resulta que se torna menor a través del tiempo, esto muestra la baja profundidad del mercado, lo cual es consecuencia de que son pocos los instrumentos que se utilizan y de manera tenue, sin aprovechar a plenitud todos los instrumentos financieros disponibles.

Se debe destacar que Chile es uno de los países que utiliza ambas fuentes de captación de recursos casi en la misma proporción, hecho que pone en evidencia un mayor conocimiento de su sector industrial y empresarial.

Cabe señalar que, para que las pymes decidan salir a bolsa deben existir mercados que hayan adoptado esta modalidad en sus operaciones, de esta manera, en Latinoamérica, según Expansión (2018), "las pymes disponen de un segmento específico en países como Brasil (BovespaMais), Perú (Mercado Alternativo de Valores) y Colombia (Segundo Mercado), mien- 
tras que otros países, como Argentina, contemplan un régimen especial que facilita su acceso al parqué" es decir, que no todos los países tienen un mercado establecido para las pymes, en algunos mercados de valores se tienen regímenes especiales o condiciones "favorables" para facilitar el acceso de estas empresas a este tipo de financiación y en otros recién se está incursionando en el mismo.

Bajo ese precedente, se debe mencionar que todavía existen barreras al acceso a financiamiento, siendo una de las más frecuentes la informalidad de las empresas, sobre todo en el sector de las pymes, a continuación, en la Tabla 4 se plasma las barreras sal financiamiento bancario bajo la perspectiva de los bancos.

Tabla 4
$\begin{aligned} & \text { Barreras de las empresas y el financiamiento bancario } \\ & \text { BARRERA }\end{aligned}$
$\begin{aligned} & \text { Falta de Información/ Informalidad } \\ & \text { Otros: }\end{aligned}$
$\begin{aligned} & \text { Bajo retorno de préstamos } \\ & \text { Baja capacidad de repago }\end{aligned}$
$\begin{aligned} & \text { Falta de interés estratégico para el banco } \\ & \text { Altos costos de transacción }\end{aligned}$
$\begin{aligned} & \text { Dificultadesejecucióncolateral } \\ & \text { Dificultad en registro de activos para colateral }\end{aligned}$
$\begin{aligned} & \text { Problemas c/personalidad legal empresa } \\ & \text { Fuente: FOMIN, VII Encuesta Regional. “TheMissingMiddle y los bancos”, 2014 }\end{aligned}$

La falta de información o informalidad es el aspecto más crítico, con un $51 \%$ sobre el total, seguido de factores como la escasa capacidad de repago de los créditos, bajo este contexto los gobiernos han procurado dar una solución, a través de la implementación de la banca de desarrollo, la cual pretende asignar recursos a aquellas PYMES que no cumplen con el perfil de la banca tradicional; sin embargo, no han sido tan exitosas como se esperaba.

Según la Asociación Latinoamericana de Instituciones Financieras para el Desarrollo (ALIDE) la importancia de la banca de desarrollo se ha incrementado en los últimos años, en países como: Argentina, Brasil, 
Costa Rica, Ecuador y República Dominicana en dónde la banca de desarrollo representa entre un 37,6\% y un 61,9\%, sin embargo aún no tienen el impacto esperado, en el resto de países como Bolivia, Colombia, Chile, Ecuador, Honduras y México, en los cuales la misma participación representa entre un $10,3 \%$ y $18,7 \%$.

Asimismo, una de las formas a través de las cuales las empresas hacen frente a su necesidad de financiamiento, es la negociación con los proveedores, este es un factor que aporta positivamente a las finanzas de las pymes, ya que los términos del plazo de pago contribuyen al logro de financiamiento de las operaciones. Es así que, de acuerdo al Instituto Iberoamericano del Mercado de Valores (IIMV), en países como Argentina, Chile, Uruguay y México, los proveedores financian a las empresas en un $3 \%, 17 \%, 15 \%$ y $14 \%$ respectivamente.

Las dos formas usuales de negociar son: el confirming y el factoring, en los cuales se gestionan los pagos a los proveedores y los cobros a los clientes, de tal forma de retrasar los pagos y anticipar los cobros, para contar con los recursos necesarios para realizar las operaciones sin inconvenientes.

En relación al factoring a continuación en la Tabla 5 se presenta el volumen de operaciones de factoring en Sudamérica.

Tabla 5

Volumen de operaciones de factoring Latinoamérica

\begin{tabular}{lccccccc}
\multicolumn{1}{c}{ País } & $\mathbf{2 0 1 1}$ & $\mathbf{2 0 1 2}$ & $\mathbf{2 0 1 3}$ & $\mathbf{2 0 1 4}$ & $\mathbf{2 0 1 5}$ & $\mathbf{2 0 1 6}$ & $\mathbf{2 0 1 7}$ \\
Argentina & 475.000 & 614.000 & 856.000 & 1.299 & 1.551 & 1.891 & 2.282 \\
Bolivia & 35.000 & 35.000 & 31.000 & 22.000 & 44.000 & 44.000 & 44.000 \\
Brasil & 45.623 & 43.627 & 31.552 & 31.782 & 28.965 & 45.379 & 50.432 \\
Chile & 21.500 & 24.000 & 25.500 & 24.850 & 22.300 & 25.050 & 22.756 \\
Colombia & 4.990 & 4.562 & 7.076 & 8.293 & 10.333 & 7.630 & 7.655 \\
Costa Rica & 30.000 & 180.000 & 115.000 & 2.337 & 2.710 & 231.000 & 231.000 \\
República Dominicana & 0.000 & 0.000 & 0.000 & 0.000 & 0.000 & 0.000 & 144.000 \\
Honduras & 0.000 & 0.000 & 0.000 & 0.000 & 22.000 & 27.000 & 27.000 \\
México & 21.074 & 26.130 & 28.061 & 25.486 & 19.291 & 22.510 & 23.314 \\
Perú & 2.461 & 2.310 & 8.163 & 8.293 & 1.475 & 155.000 & 10.105 \\
Uruguay & 0.000 & 61.000 & 58.000 & 70.000 & 90.000 & 84.000 & 98.000 \\
Total & 98.199 & 103.531 & 103.425 & 105.138 & 88.796 & 105.017 & 119.105 \\
\hline
\end{tabular}

Fuente: Factors Chain International (FCI) 
Se puede observar que países como Brasil, Chile, México y Colombia hacen uso de esta estrategia como una forma de conseguir recursos para las operaciones de las empresas, sin embargo, países como Bolivia, Honduras, República Dominicana e incluso Uruguay aún no se han introducido de manera más intensa en el factoring, que llega a ser una alternativa atractiva.

\subsection{Razones por las cuales no se utiliza el mercado de valores}

A continuación, se analizan las razones por las cuales las pymes no utilizan con frecuencia al mercado de valores como alternativa al financiamiento.

\subsubsection{Empresas familiares}

En Latinoamérica existen empresarios dispuestos a realizar emprendimientos, cada uno de ellos en distintos rubros y con diferentes características, esto hace que exista una diversidad de pymes que logran ingresar al mercado o mantenerse en él, lo cual depende de muchos factores, encontrándose entre ellos la disponibilidad de recursos.

En tal sentido, una empresa en Latinoamérica usualmente para financiarse utiliza recursos propios, tal cual indican las tres "F", "Family, Friend and Fool", es decir, familia, amigos y osados que se atreven a invertir en un proyecto o idea, y esto funciona de la misma manera en cualquier parte del mundo.

En efecto, un gran número de empresas surgen en el seno de la familia, algo característico en Latinoamérica, dado que según FORBES, el 70\% de las pymes en Latinoamérica son familiares ${ }^{8}$.

Estas han sido y son en gran parte administradas bajo la experiencia empírica de los dueños fundadores con una visión familiar, sin embargo, ante el crecimiento del negocio se necesita de personal profesional que determine procesos tanto administrativos, financieros y comerciales, que sean más técnicos y apropiados para la producción industrial y en serie.

Una vez que la empresa empieza a crecer, también lo hacen sus necesidades de ampliar el capital, para ello existen dos alternativas, realizar una

8 https://www.forbes.com.mx/pymes-en-latinoamerica-empresa-familiar/ 
Oferta Pública de Venta $\left(\mathrm{OPV}^{9}\right)$ a través de una salida a bolsa o una emisión de deuda, en el caso de financiarse mediante el mercado de valores.

En el primer escenario, se ve que existe un escepticismo por despegarse del total del capital accionario, no solamente en América Latina sino en cualquier parte del mundo, es decir, una salida a bolsa implica la entrada de nuevos inversionistas a la empresa, y como las bolsas de valores por lo general imponen niveles mínimos de free float ${ }^{10}$, por temas de liquidez del mercado y dinamismo en las negociaciones, significa que las empresas familiares al no tener el 100\% de la participación, deberán ceder parte del control de su empresa y por supuesto esto causa susceptibilidades.

Sin embargo, la participación en el mercado de valores por parte de este tipo de empresas podría ser mejor aprovechada, pues muchos inversionistas decidirán invertir en ellas, debido a que la característica principal es que el dueño tiene pleno conocimiento del negocio, de cómo funciona en sus aspectos operativos, administrativos, financieros, etc., entonces, al tener el "knowhow" del negocio, el inversionista está motivado a invertir, ya que este hecho si bien no asegura el éxito del negocio, pues al menos genera mayor confianza en el crecimiento del mismo.

Por lo tanto, una de las razones por las cuales los empresarios no utilizan el mercado de valores, según Peña (2018) es un miedo a una posible pérdida de control en el caso de dar entrada a nuevos accionistas, y posteriormente no tener toda la potestad de tomar las decisiones respecto al futuro de sus empresas; decisiones como expandirse, innovar, etc., son aspectos que no se quiere dejar en manos de terceros.

\subsubsection{Estructura financiera empresarial}

Tanto en pymes como en grandes empresas, sea que coticen o no en el mercado bursátil pero decidan salir a él, la estructura financiera es un aspecto determinante y esto influye totalmente en las operaciones de cada gestión, sin embargo muchos empresarios piensan que al adquirir deuda, están hipotecando las operaciones empresariales y perdiendo presencia en el mercado, pero es preciso mencionar que los problemas de las em-

9 Oferta Pública de Venta de acciones, es una operación financiera que se realiza con el fin de vender una parte o la totalidad del capital social de una empresa al público en general o a inversores institucionales.

10 Porcentaje de acciones disponibles para el público 
presas no recaen justamente en la deuda, sino de la capacidad de estas de generar caja para hacer frente a sus obligaciones con terceros.

Existen entonces dos escenarios en cuanto a la estructura financiera, el primero de ellos responde al uso de recursos propios con mayor porcentaje dentro de la estructura, esto puede ser el capital inicial, ampliaciones de capital, las reservas u otros, de acuerdo a esto, se puede llegar a tener rentabilidad inferior a la que se tendría en caso de utilizar deuda con terceros.

El segundo escenario sería la emisión de deuda en mayor proporción que los recursos propios, que puede ser adquirida a través de créditos bancarios, emisión de bonos, crowdlending, etc., en este caso se puede obtener mayor rentabilidad, pero para ello se debe tener en cuenta el ciclo de negocio de la empresa.

Específicamente se requiere de generación de caja durante una gestión, además de la liquidez que es capaz de generar la empresa, para tener recursos disponibles de manera constante para financiar las operaciones de la misma. Por su puesto para esto se debe realizar una planificación estratégica de las gestiones que se realizaran durante un periodo de tiempo y de los recursos necesarios para lograr los objetivos establecidos a futuro.

En dicha planificación, se debe considerar la estructura financiera que se manejará en función de las necesidades de cada empresa y del sector en el que se desenvuelva, por lo tanto, se deberá evaluar la conveniencia y pertinencia de que una empresa se financie a través de la salida a bolsa o de la emisión de deuda.

Para ello, es fundamental conocer la empresa y hacer una evaluación de sus necesidades para que se obtenga la estructura más conveniente para la misma, de tal forma que pueda hacer frente a las obligaciones posteriores, bajo condiciones favorables en comparación de las otras formas de financiamiento, esto es algo que las empresas no están acostumbradas a hacer, es decir buscan financiamiento y lo obtienen según lo que hallan en el mercado, pero no muchas efectúan un análisis profundo de lo que realmente necesitan, es por ello que los resultados no son lo que se esperaban. 
Es así que, otra razón por la cual un empresario decide no acudir a la bolsa de valores, es que no realiza el análisis pertinente respecto de la estructura financiera que la empresa requiere en función de sus capacidades, objetivos y sector.

\subsection{Ventajas de financiarse a través de la bolsa de valores}

Dentro de la estructura financiera de las empresas, siempre ha existido una alternativa latente al financiamiento bancario, que es la bolsa de valores, sin embargo, no siempre las pymes han podido acceder a este mercado por las razones mencionadas anteriormente, es por esto que surge la pregunta ¿por qué deberían las pymes acudir a la bolsa o a su mercado alternativo para financiarse?

Según Expansión (2018), salir a Bolsa permite obtener financiación sin elevar el endeudamiento y además aporta protagonismo entre clientes y proveedores en el mercado. Sin embargo, también implica costos económicos importantes y la obligación de cumplir con diversos requisitos legales y de transparencia para con la bolsa y el mercado.

Otra gran ventaja de la cotización en un mercado bursátil para pymes como indica Cepymes (2018) es la exposición que estas empresas llegan a tener a nivel internacional. De esta manera esto permite que inversores de todo el mundo sigan de cerca la evolución de estos mercados, con la finalidad de posteriormente poder tomar parte en las compañías a través de la inyección de capital. Los mercados de valores sirven de trampolín a las empresas que tienen como objetivo la expansión a otros países.

En base a esto, se argumenta que existen dos razones fundamentales para que estas empresas consideren al mercado de valores como una alternativa estratégica para acceder a financiación por terceros, las cuales se mencionan a continuación:

\subsubsection{Transparencia}

Una de las razones fundamentales es la transparencia que adquieren las pymes al salir a bolsa o al utilizar la bolsa como medio de financiamiento, dado que la información deja de ser privada y se pone a disposición 
del público en general, siendo este uno de los requisitos implícitos de participar en el mercado.

Tal y como indica Cepymes (2018) la salida a bolsa para las pymes incrementa la reputación, credibilidad y visibilidad empresarial, esto se da sobre todo a la hora de conseguir nuevos clientes o también proveedores, especialmente en entornos internacionales.

De esta manera, la publicación de sus estados financieros, el conocimiento de los objetivos de largo plazo, sus estrategias, etc., hacen que la empresa refleje una imagen de seriedad y solidez, lo cual la hace más atractiva y confiable ante los inversionistas.

Si el objetivo es captar más recursos, es necesario a mediano y largo plazo transparentar el manejo de la empresa, a fin de cumplir con los requisitos, normativa y estándares establecidos en las diferentes bolsas de valores.

Lo contrario sucede con un crédito tradicional (para nada desmereciendo esta alternativa de financiamiento), dado que quien tiene que analizar los estados financieros y la generación de caja de la empresa es la institución bancaria, y posterior al análisis decide si es viable o no la proporción de financiamiento.

Se debe hacer notar que, para lograr una transparencia efectiva, es necesario un cambio estructural en el manejo de la empresa, respecto a su visión, misión, administración y gestión, para que así se atiendan a los requerimientos de fluidez de información en tiempo real.

\subsubsection{Condiciones}

Según Expansión (2018), las fuentes de financiación cambian a lo largo de la evolución natural de una empresa. En primera instancia, se recurre a la financiación de conocidos o familiares, luego a la bancaria, posteriormente al capital riesgo y llega un momento en el que, si las pymes llegan a tener un plan de expansión muy definido, optan por recurrir a los mercados de valores, el cual tiene condiciones específicas.

Las ventajas que tienen las pymes al ingresar a un mercado de valores, son las condiciones bajo las cuales puede salir a bolsa o emitir deuda, 
las mismas son más favorables y atractivas respecto a la banca tradicional, en conceptos tales como: las garantías, las tasa de interés, plazos, montos, etc., no obstante hay que mencionar que no en todos los mercados esto realmente es una ventaja, puesto que algunos de ellos no le otorgan un beneficio realmente diferenciador a la empresa; esto depende de la bolsa, y el desarrollo de su mercado con enfoque a este sector empresarial.

Para las pymes, representa una ventaja poder acceder a un mercado en el cual los inversionistas han de poner capital en su empresa por la mera convicción de un proyecto de inversión, o la seguridad de la devolución de capital. Esta no tiene comparación con un mercado tradicional de crédito bancario.

El mercado de valores debería ser considerado con mayor énfasis, por las empresas en los países que realmente se hace un tratamiento significativo en ello, uno de los mercados que plantea una ventaja interesante es Perú, a través de su Mercado Alternativo de Valores (MAV), donde se plantean adecuadamente las bases, los requisitos reducidos en comparación con el mercado principal y ventajas posteriores de poder acceder a un mercado principal directamente.

Otros países en Latinoamérica, no plantean ideas concretas sobre un mercado alternativo de PYMES, tal es el caso de Bolivia o Argentina, incluso Chile, que ha sido uno de los países con mayor estabilidad en el ámbito financiero.

\subsection{Educación financiera}

Es evidente que han existido corrientes sobre educación financiera, aquello que se entiende como una capacitación, sobre todo a jóvenes, sobre términos y procedimientos financieros como el ahorro, la inversión y el financiamiento, sin embargo, en países en los que aún no se tiene un desarrollo completo del mercado de valores, la gente, por lo general no conoce lo que es un mercado de valores como tal, ni su procedimiento y mucho menos sus fines.

En términos generales, la cultura financiera bursátil puede ser adquirida mediante la propia educación que imparten los bancos centrales o las entidades reguladoras de los sistemas financieros, o a través de la 
misma sociedad, de esta manera uno podría empaparse de la existencia de un mercado y su comportamiento, o crecer con él, es decir, conocer el comportamiento del mercado día a día, los índices bursátiles, la cotización empresarial, etc. Esas últimas sociedades, son las que crecen con más fuerza en el aspecto financiero, el caso de Latinoamérica, algunos países como Colombia, Perú, Chile, y México, son los que han tomado conciencia de que se debe facilitar la educación financiera de manera más intensa, de tal forma que sea parte de la vida diaria de la población, para que lograr el desarrollo del mercado de valores.

Es por ello que, es necesario estructurar un programa de educación financiera a nivel nacional en cada país de Latinoamérica, para contribuir con la alfabetización financiera y demás aspectos relacionados a la educación, que posteriormente tendrán frutos, este es un aspecto fundamental que las bolsas y los organismos públicos gubernamentales deberían tomar en cuenta como parte de una profundización en los mercados.

El tema base es la concientización, es decir, hay países en Latinoamérica que no desarrollan a cabalidad la formación financiera bursátil en su población, de esta manera, para un emprendedor, que ha crecido con su empresa, intentar introducir a otros inversionistas en ella resulta un aspecto poco atractivo.

Es por eso, que las pymes en su mayoría empresas familiares, que han ido desarrollando sus actividades de manera "familiar" tienen una cultura bursátil, cerrada sin indicios de querer dar paso a un mercado alternativo fuera de lo tradicional, el cual de cierta forma impulsaría con mayor énfasis su crecimiento.

Ni la transparencia ni los requisitos, para muchos representan alternativas atractivas, de esta manera, es que las pymes en la región necesitan un impulso con mayor énfasis en el desarrollo post bolsa, y para ello se necesitan de mayores beneficios y políticas gubernamentales que apoyen la financiación a través del mercado de valores.

Concientizarlos bursátilmente, hacer conocer a detalle los beneficios que estas otorgan a largo plazo, es una de las maneras entre muchas para que las pymes consideren realmente a ésta como alternativa. 
Es por esto, que en respuesta a la necesidad de una concienciación de la importancia del acceso y uso del mercado de valores para lograr el financiamiento de las empresas y sobre todo de las pymes, surge la necesidad de educación financiera bursátil.

Si bien los actores del mercado de valores, tales como: los emisores, inversionistas, calificadoras de riesgo, sociedades titularizados y reguladores requieren de educación bursátil; también se debe tener en cuenta la formación del empresario, esta deberá enfocarse en la norma que rige el mercado de valores en cada uno de los países; cómo se realiza una emisión, los requisitos que deben presentarse, el funcionamiento del mercado, las consecuencias, los riesgos, pero sobre todo las ventajas que se presentan al participar en el mismo. Además, debe ser coordinada con las instancias que estén vinculadas para difundir información verídica, integral y estructural respecto del mercado de valores.

\section{Conclusión}

La importancia de las pymes en Latinoamérica, principalmente radica en el hecho de que generan el 61\% del empleo en la región y la necesidad de crecimiento se refleja en la participación del 15\% en la producción, es decir las empresas no son productivas, y uno de los factores principales que no permite el desarrollo de las mismas, es el financiamiento.

El financiamiento puede ser interno o externo, y el mismo debe ser resultado de un análisis de la estructura de capital de le empresa, y de esta manera definir la fuente de recursos menos costosa y más adecuada, siendo la más tradicional la banca. Al respecto, las principales causas por las cuales las empresas no acceden a financiamiento se deben a: las elevadas tasas de interés, la falta de acceso a créditos de largo plazo, y las dificultades asociadas a la constitución de garantías, y como respuesta a las mismas, los gobiernos han implementado la banca de desarrollo, y las empresas han implementado formas de gestión de recursos con sus proveedores, sin embargo, esto no parece ser suficiente para cubrir las necesidades de las pymes.

Es por ello, que como alternativa se propone la incursión en el mercado de valores, la cual tendría beneficios tales como: la transparencia 
del manejo de la empresa y la publicación de información de la misma; y las condiciones favorables encontrándose entre ellas, la tasa de interés, garantías, y plazo principalmente; sin embargo, cabe señalar que las condiciones varían, dependiendo del país de la región.

Estos aspectos deben ir acompañados de la educación financiera, ya que la falta de conocimiento de la existencia del mercado de valores, de los diferentes instrumentos disponibles y de su funcionamiento, limitan el uso de la misma para que las empresas logren el financiamiento de sus actividades.

Se observó que, en Latinoamérica no se utiliza el mercado de valores de manera profunda, pero se están haciendo intentos para intensificar su uso, el más notable hasta el momento es la creación del MILA, que es la integración de las bolsas de valores de México, Perú, Colombia y Chile. Este hecho pone en evidencia que, en la región existe potencial para intensificar el acceso al mercado de valores, lo cual tendría como efecto el impulso de las empresas para su desarrollo y crecimiento, siendo el objetivo alcanzar la exportación de sus bienes o servicios, para ser competitivas a nivel mundial.

Cuando una empresa se establece, el primer beneficio es la generación de empleos, lo cual contribuye a que las familias perciban ingresos y se fomente el consumo y por ende la producción, fomentándose el crecimiento de la economía del país, en este hecho radica la importancia de que las pymes consigan el financiamiento para realizar sus operaciones.

Adicionalmente, se debe considerar el hecho de que el resto del mundo utiliza esta herramienta como una de las fuentes principales de recursos, por supuesto, Latinoamérica debe ajustar las condiciones a sus características y necesidades, pero no por ello debe dejar de lado una herramienta tan poderosa que funciona en el resto del mundo y que podría ser la respuesta a los requerimientos de capital para que las empresas Latinoamericanas amplíen su mercado y mejoren su productividad.

Es por ello que, del análisis realizado se concluye que una alternativa al financiamiento bancario de las empresas sería el mercado de valores; sin embargo, el mismo no está siendo utilizado plenamente debido a que las pymes en Latinoamérica son principalmente empresas familiares, 
pero además porque no se realiza una evaluación prudente respecto de la estructura financiera adecuada, para cada empresa según sus características, mercado y objetivos.

Por los aspectos observados, para fomentar el uso del mercado de valores en Latinoamérica, se propone un programa de educación financiera, enfocado principalmente al sector empresarial, y las instituciones vinculadas con sus operaciones, para que las empresas tomen conciencia de la importancia y potencial del mercado de valores, para así puedan hacer uso de la misma.

\section{Referencias Bibliográficas}

AGENCIA EFE (2017). Las pymes latinoamericanas aportan solo el $30 \%$ del PIB regional, México. p.1.

Álvarez, Iván (2016). Finanzas estratégicas y creación de valor. Bogotá: ECOE Ediciones: 486 p.

Asociación Latinoamericana de Instituciones Financieras para el DesarrolloALIDE (2015). Desempeño de la Banca de Desarrollo Latinoamericana, Iniciativas Regionales y una visión Global de la Financiación. 93 p.

Bolsa de Valores de Colombia - BVC (2014). Guía del Mercado de Valores. 224 p.

CAIXABANK (2012). Manual de la inversión en bolsa. Madrid: Cobrhi, S.L. 143 p.

CEPYMENEWS (2018). El crecimiento del sector pyme a través de la bolsa. Opinión. España. p1.

Consultive GrouptoAssistthe Poor - CGAP (2010). Financial Access 2010, The State of Financial Inclusion Throughthe Crisis.106 p.

Comisión Económica para América Latina y el Caribe-CEPAL (2018). MIPYMES en América Latina. Un frágil desempeño y nuevos desafíos de las políticas de fomento.557 $\mathrm{p}$.

Comisión Económica para América Latina y el Caribe-CEPAL (2010). Crisis originada en el centro y recuperación impulsada por las economías emergentes, Panorama de la inserción internacional de América Latina y el Caribe, Documento Informativo 2009-2010.216 p.

Comisión Económica para América Latina y el Caribe - CEPAL/Banco Interamericano de Desarrollo - BID/Organización de los Estados Americanos - OEA 
(2011). Experiencias exitosas en innovación, inserción internacional e inclusión social: Una mirada desde las PyMES.113 p.

Expansión (2017). Cómo escoger el mejor mercado para salir a bolsa, España, 2 p.

Federación Iberoamericana de Bolsas - FIAB (2019).Anuario Estadístico Fact Book 2018. 55p.

FORBES (2017). Pymes en Latinoamérica, ¿empresa familiar?. México. p1.

Heras, José (2001). Diccionario de mercados financieros. Barcelona: Gestión 2000. $180 \mathrm{p}$.

Galán, Liliana.; De Giuste, Eduardo.;Mollo, Gabriela y Nóbile, Cecilia (2007). La Problemática del financiamiento en el sector PyME - Región Este de la prov. de Buenos Aires. Argentina. 26p.

Instituto Iberoamericano de Mercado de Valores - IIMV (2017). Estudio sobre la financiación de las micro, pequeñas y medianas empresas a través de los mercados de capitales en Iberoamérica. Madrid: CYAN, Proyecto Editoriales, S.A.282 p.

Llisteri, Juan; Vivanco, Fermín; Ariano, María (2002). Acceso de las pequeñas y medianas empresas al financiamiento allá de las fronteras. Washington, D.C. 76 p.

Opertti, Fabrizio (2017). Conoce por qué para el BID las PYMEs de América Latina y el Caribe están formadas por héroes y heroínas. Washington, D.C. p1.

Peña, Jesús (2018). La Bolsa para pymes, el MAB, una fuente de financiación. España. p1.

Rojas, Laura (2017). Situación del financiamiento a PYMEs y empresas nuevas en América Latina. Chile: Gráfica LOM. 62 p.

Saavedra, María (2014). Latinoamérica. La problemática del financiamiento. México, p. 1-5.

Standard \&Poors Dow Jones Índices - S\&P (2016) S\&P MILA Índices Metodología. $30 \mathrm{p}$.

Van Den Verghe, Edgar (2015). Diseñe y Administre su propia empresa. Bogotá: ECOE Ediciones. 375 p. 